\title{
Semiconductor gas sensing coupled with pre-sampling system for public security
}

\author{
Valeriy V. Krivetskiy ${ }^{1}$, Maxim A. Panteleev ${ }^{1}$, Leonid A. Logvin ${ }^{1}$, Andrey V. Smirnov ${ }^{1}$, Marina N. \\ Rumyantseva ${ }^{1}$, Sergey Yu. Semenov', Valentin N. Smirnov', Alexander M. Gaskov ${ }^{1}$ \\ ${ }^{1}$ Department of Chemistry, Lomonosov Moscow State University, Leninskie Gory 1-3, Moscow, \\ Russia, 119991, \\ vkrivetsky@gmail.com \\ ${ }^{2}$ Research and Technical Center of Radiation-Chemical Safety and Hygiene FMBA Russia, \\ Shchukinskaya st., 40, Moscow, Russia, 123182
}

\begin{abstract}
An innovative approach of sensitive and selective detection of trace amounts of chemical threat agents is presented in this work. The technology deals with semiconductor metal oxide gas sensors as a means of express gas detection, coupled with pre-sampling system, which is to improve sensitivity and selectivity of analysis. Examples for $\mathrm{H}_{2} \mathrm{~S}$, DMMP (phosphor-organic weapons simulant) and UDMH (highly toxic rocket engine fuel constituent) sensor detection with aided by pre-sampling system are given. The issues of sensor and adsorbent materials selection and their working parameters are discussed.
\end{abstract}

Key words: semiconductor, gas sensor, sensitivity, selectivity, public security, pre-sampling

\section{Introduction}

Numerous chemical threat agents, such as explosives, super toxic compounds and chemical warfare, bear a great danger to the population due to terrorist activity, technological failures or even disasters. For the public security sake they are to be detected in the atmospheric air usually in extremely low concentrations in the express way. The general solution for problem of air quality assessment, a mass spectrometry apparatus coupled with gas chromatography, could not be applied for the described task due to a list of reasons - it is not suitable for continuous analysis in an express mode in a field conditions and usually requires numerous additional equipment which makes analysis prohibitively costly. That is the reason, why air quality analysis by means of sensor systems attracts much attention. However the task of reliable detection of extra low concentrations (below 1 ppm level) of chemicals in air could not be solved even by metal oxide semiconductor gas sensors - one of the most sensitive type of sensors. The problem becomes even more complicated when the target substance is to be detected in the presence of moisture or other air pollutants, such as perfumes, car fuel vapors etc. Neither single sensor devices nor complex sensor arrays usually referred to as "electronic noses" are able to solve the problem with demanded accuracy.

We propose that the task of express detection of extra low concentrations of air impurities with desired sensitivity and selectivity could be worked out by sensor systems when assisted by pre-sampling system. Pre-sampling consists of two successive stages: adsorption of target molecules from the air flow on specific adsorbent and then desorption during the fast heating.

Such gas analysis system, designed for detection of certain chemicals in certain background, should include a matching sensor or a combination of sensors, possessing as high selectivity and sensitivity as possible and a pre-sampling system, which is designed to fulfill the deficient parameters of sensor system.

\section{Sensor materials design}

One of the most versatile and fruitful technique of metal oxide resistive type semiconductor gas sensor selectivity improvement to the date utilizes the principle of adjustment of the type and concentration of active centers on the surface of sensor material which are responsible for solid-gas chemical interaction [1]. To achieve this adjustment nanocrystalline $\mathrm{SnO}_{2}$, which is used as a sensor material most often, undergoes surface chemical modification 
procedure. Recently it was experimentally shown that a number of synthetic concepts could be borrowed from the field of heterogeneous catalysis in order to obtain sensor material with desired working parameters [2, 3]. Such concepts are based on a huge amount of empirical data and allow bypass to some extent the step of gas-sensor interaction mechanism investigation. Though the knowledge of this mechanism could be highly useful for design of materials with high selectivity and sensitivity, modern analytical methods are still lacking capacity to reveal details of solid-gas chemical interactions especially in the case of metal oxides. The mentioned concepts deal with metal oxide acidbase and Red/Ox parameters as a measures of their chemical activity during the steps of gas molecules adsorption and conversion [4-6]. These parameters are calculated on the basis of materials structure and chemical composition. This way a number of highly selective and sensitive materials has been obtained to the date and it can be stated that there is still a plenty room for further improvements [7].

\section{Pre-sampling materials design}

The role of pre-sampling procedure in express gas analysis can be relatively divided into three adsorption of target substances, sufficient adsorption capacity, evolution of adsorbed molecules in acceptable temperature range (usually up to $400^{\circ} \mathrm{C}$ ) without decomposition or with transformation into desirable substances, invariability of adsorption properties during many consecutive adsorption/thermodesorption cycles [].

\section{Application of combined pre-sampling and sensor detecting system}

Several examples of application of different presampling materials with semiconductor gas sensors are presented below.

For $\mathrm{H}_{2} \mathrm{~S}$ concentration the Al-containing MCM41 type mesoporous material was used. To increase the number of adsorption sites the sample was additionally modified by introduction of alumina into the pores of $\mathrm{Al}$ MCM-41. Hydrogen sulfide detection using $\mathrm{SnO}_{2}$-based sensor with pre-sampling procedure aids somewhat 5-fold increase in sensor response compared to detection without pre-sampling (Fig. 1). Pre-sampling conditions was as follows: 6 minutes $\mathrm{H}_{2} \mathrm{~S}$ adsorption from air flow, than 6 minutes desorption during fast heating of the sorbent $\left(250{ }^{\circ} \mathrm{C} / \mathrm{min}\right)$ up to 300 ${ }^{\circ} \mathrm{C}$ and keeping at this temperature; after that pre-sampling procedure was repeated.
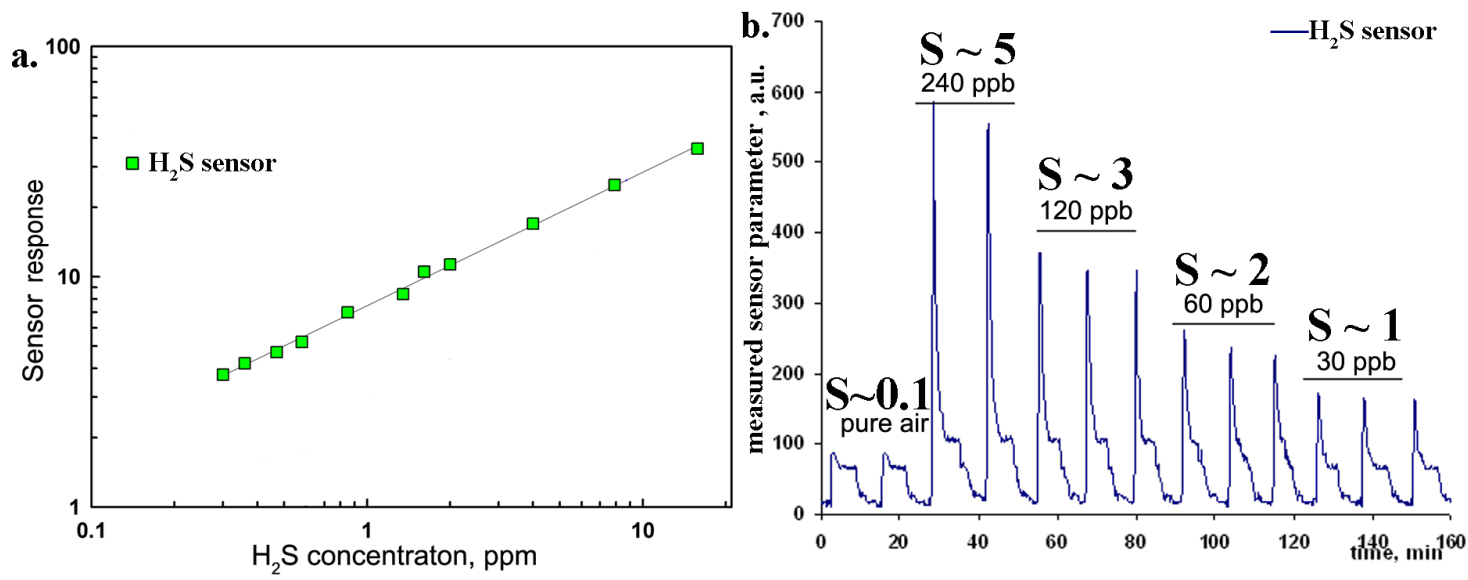

Fig. 1. $\mathrm{H}_{2} \mathrm{~S}$ sensor response in the $0.3-11 \mathrm{ppm}$ range without pre-concentration (a) and $0.03-0.24 \mathrm{ppm}$ with pre-concentration (b).Sensor response was calculated according to formula $S=\Delta G / G_{0}$, where $G_{0}$ - conductance of sensor material in pure air flow, $\Delta G$ - conductance change during exposure to tested gas flow.

different functions: concentration, separation and conversion. In some cases sorbent can execute two or even all these functions.

The application of pre-sampling for concentration purposes results in instantaneous increase of concentration of substance to be determined in the gas flow which is detected by sensor system response has as a peak shape. Sorbents used for pre-sampling should possess the following main properties: specific selective
Next example demonstrates the case where pre-sampling sorbent bears two functions concentration and separation. The target substance was dimetyl methylphosphonate (DMMP), widely used as a simulant of phosphorus-based threat agents. One of the major problems of sensor detection of such kind of complex compounds is a strong interfering effect with other classes of substances, in particular, alcohols. 


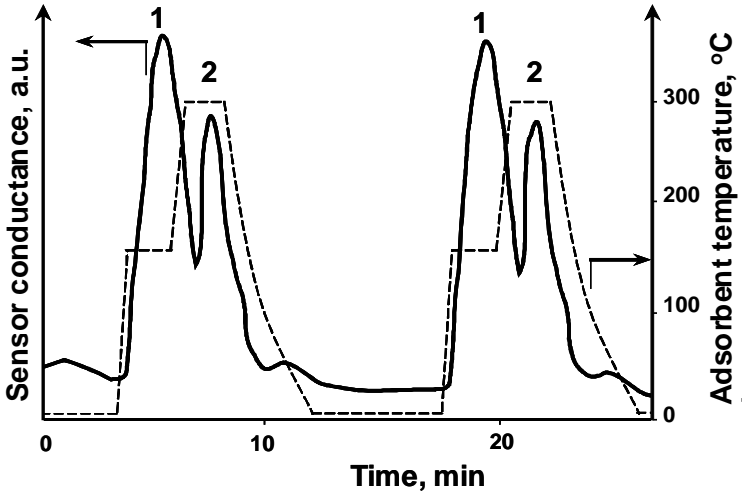

Fig. 2 Pre-sampling for separation of target component DMMP (2) from masking compound ethanol (1).

a)

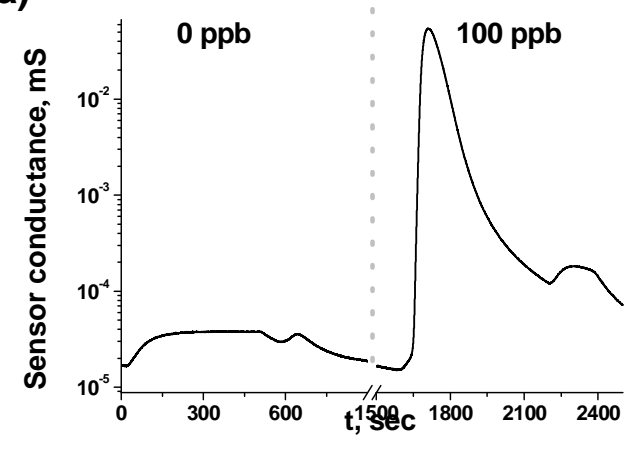

The third example exhibits the conversion mode of pre-sampling system, which is very promising route for the improvement of sensitivity and selectivity of the sensor detecting system. The idea is to use a concentrator along with the catalyst for selective transformation of target compound into another compound, which can be determined by sensors more selectively and more precisely than initial one. Fig. 3 presents data on detection of unsymmetrical dimethyl hydrazine (UDMH) in air through its conversion to nitrogen dioxide. Sensor determination of nitrogen dioxide is significantly more selective then UDMH since $\mathrm{NO}_{2}$ is the one oxidant gas and is easily detected even in low amounts in the presence of any kind of reducing gas. Fig. 4

b)

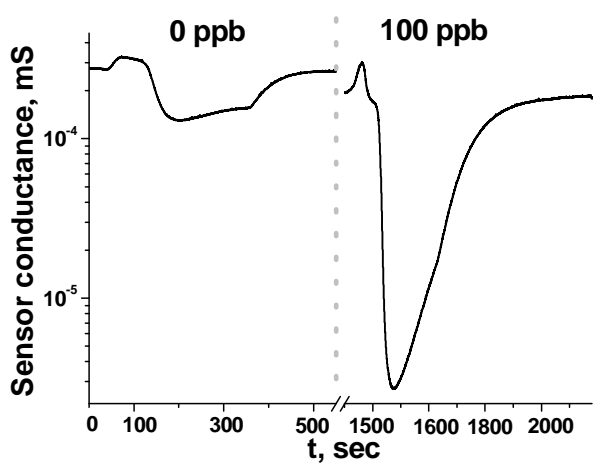

Fig. 3 Pre-concentration of UDMH (100 ppb) on inert adsorbent (a) and pre-concentration on active adsorbent, with further conversion to $\mathrm{NO}_{2}(b)$.

The application of specific sorbent along with special thermodesorption regime decreases this effect in a considerable degree. Silica based material was used as a concentrator; model air flow contained $640 \mathrm{ppb}$ of DMMP and $1 \%$ of ethanol. It was found, that adsorption of DMMP on this type of sorbent was noticeably stronger that of ethanol. Thus, the thermodesorption of ethanol during the temperature rise occurred before DMMP thermodesorption (Fig. 2). In order to use this effect for separation of two compounds and their independent detection, the temperature program during desorption stage consisted in two steps: fast heating up to $150^{\circ} \mathrm{C}$ with 2 minutes plateau at this temperature and, finally, heating up to $300{ }^{\circ} \mathrm{C}$. The peak 1 on the curve of sensor signal corresponds to ethanol evolution while the peak 2 to DMMP desorption. This example shows potential possibility to increase selectivity of detecting system using pre-sampling procedure with specific concentrators and separators. This approach was also successfully applied for DMMP detection in the presence of other interfering components, such as methanol, water and acetone. shows concentration dependence of sensor response to UDMH being measured through preconcentration, coupled with conversion to $\mathrm{NO}_{2}$.

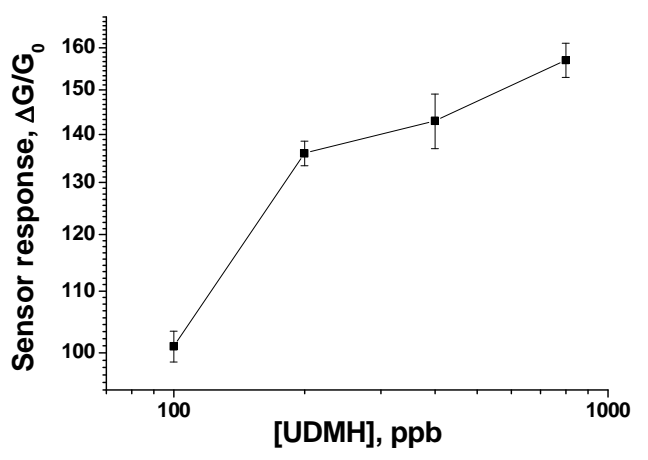

Fig. 4 concentration dependence of sensor response to UDMH being measured through preconcentration, coupled with conversion to $\mathrm{NO}_{2}$.

Silica based concentrating sorbent with deposited catalytically active metal oxide phase was used as the pre-sampling material. Experiment was carried out at the following 
conditions: consumption of UDMH (100-1000 $\mathrm{ppb}$ ) from air flow within 5 minutes and then UDMH conversion to $\mathrm{NO}_{2}$ during the fast heating in air flow to $300^{\circ} \mathrm{C}$. As a result, strong response to $\mathrm{NO}_{2}$ was observed.

\section{Conclusions}

Pre-sampling system is a powerful tool for mitigation of the drawbacks of semiconductor based sensor systems for express gas analysis. It does not increase the time of a single analysis cycle considerably while effectively rises both test sensitivity and selectivity. Provided examples show the potential for application of such systems for detection of hazardous gases below ppm level which is critical to provide public security in many circumstances.

\section{Acknowledgements}

The work was supported by The Ministry of Education and Science of Russian Federation state contract 11.519.11.1009.

\section{References}

[1] M. N. Rumyantseva and A. M. Gas'kov, Chemical modification of nanocrystalline metal oxides: effect of the real structure and surface chemistry on the sensor properties, Rus. Chem.I Bull. 57, 1106-1125 (2008); doi:10.1007/s11172-0080139-z

[2] V.V. Kovalenko, A.A. Zhukova, M.N. Rumyantseva, A.M. Gaskov, V.V. Yushchenko, I.I. Ivanova and T. Pagnier, Surface chemistry of nanocrystalline $\mathrm{SnO}_{2}$ : Effect of thermal treatment and additives, Sens. Act. B: Chem. 126, 52-55 (2007); doi: 10.1016/j.snb.2006.10.047,

[3] V. Krivetskiy, M. Rumyantseva, A. Gaskov, Design, Synthesis and Application of Metal Oxide Based Sensing Elements: A Chemical Principles Approach in "Metal Oxide Nanomaterials for Chemical Sensors" edited by Chris Balmes, Springer Science+Business Media, to be published in 2012

[4] P. Moriceau, A. Lebouteiller, E. Bordes and P. Courtine, A new concept related to selectivity in mild oxidation catalysis of hydrocarbons: the optical basicity of catalyst oxygen, Phys. Chem. Chem. Phys. 1, 5735-5744 (1999); doi: 10.1039/A908314F

[5] M. Henry, Partial charges distributions in crystalline materials through electronegativity equalization, Materials science forum 152-153, 355-358 (1994)

[6] A. Bielański, J. Haber, Oxygen in Catalysis on Transition Metal Oxides, Catalysis Reviews 19, 141 (1979); doi: 10.1080/03602457908065099

[7] V. Krivetskiy, A. Ponzoni, E. Comini, S. Badalyan, M. Rumyantseva, A. Gaskov, Selectivity Modification of $\mathrm{SnO}_{2}$-Based Materials for Gas
Sensor Arrays, Electroanalysis 22, 2809-2816 (2010); doi:10.1002/elan.201000277

[8] Leonid A. Logvin, , Andrey V. Smirnov, Roman A. Ovsyannikov, Vitaly V. Ordomsky, Marina N. Rumyantseva, Alexander M. Gaskov, Francois Fajula, Irina I. Ivanova, Pre-concentration of organophosphorous compounds on porous silica materials, Studies in Surface Science and Catalysis 174, 623-626 (2008); doi: 10.1016/S0167-2991(08)80277-7 